EXTENDED REPORT

\title{
Corneal stromal changes following reconstruction by ex vivo expanded limbal epithelial cells in rabbits with total limbal stem cell deficiency
}

\author{
E M Espana, S-E Ti, M Grueterich, A Touhami, S C G Tseng
}

Br J Ophthalmol 2003;87:1509-1514

See end of article for authors' affiliations

.....................

Correspondence to: Scheffer C G Tseng, MD, $\mathrm{PhD}$, Ocular Surface Center, 7000 SW 97

Avenue, Suite 213, Miami, FL 33173, USA;

stseng@ocularsurface.com

Accepted for publication 3 July 2003

\begin{abstract}
Aim: To study corneal stromal changes and the presence of myofibroblasts after transplantation of ex vivo expanded limbal epithelium.

Methods: A state of limbal deficiency was induced in 16 rabbits. After transplantation with autologous ex vivo expanded limbal epithelium on amniotic membrane (AM), their clinical outcomes were classified as success, partial success or failure according to surface smoothness, stromal clarity, and vascularisation. Clinical outcomes were correlated with phenotypic outcomes of corneal, conjunctival, or mixed epithelium, defined by expression of K3 keratin or MUC5AC. Immunostaining was performed with antibodies against collagen IV, fibronectin, and $\alpha$-smooth muscle actin $(\alpha-S M A)$ to assess stromal wound remodelling. Results: Rabbits were sacrificed after a mean follow up of 10 (SD 3.3) months. Collagen IV, expressed in the basement membrane of all three groups, was found in the stroma of the partial success, but not in that of the success or the failure. Fibronectin was absent in the success and the failure, but expressed in the stroma of the partial success. $\alpha$-SMA was expressed in superficial stroma of the partial success, but suppressed in areas with $A M$ remnants.

Conclusion: Restoration of a clear and transparent cornea is associated with a normal corneal epithelium and complete wound remodelling. In contrast, wound healing remains active and incomplete in conjunctivalised corneas, which remain opaque with myofibroblasts.
\end{abstract}

O ne major advance made in treating patients suffering from total limbal stem cell deficiency is to transplant epithelial cells that have been expanded from a small limbal biopsy cultured on amniotic membrane. ${ }^{1-5}$ We have recently demonstrated the efficacy of this new surgical procedure in a rabbit model of unilateral total limbal stem cell deficiency. ${ }^{6}$ After more than 1 year follow up, we noted that the control group receiving amniotic membrane transplantation (AMT) alone showed $100 \% \quad(n=10)$ clinical failure with vascularised and scarred corneas. In contrast, AMT with ex vivo expanded limbal epithelial cells showed $26 \%(11 / 42)$ clinical success and 19\% (8/42) partial success (both $(\mathrm{p}<0.0001)$, resulting in total and partial recovery of a smooth, avascular, and clear cornea, respectively. ${ }^{6}$ Furthermore, clinical success is correlated with the recovery of a normal corneal epithelial phenotype $(\mathrm{p}<0.05)$, and clinical failure is correlated with the maintenance of a conjunctival epithelial phenotype $(\mathrm{p}<0.005)$ ( Ti et al, submitted, 2003).

During the above investigation, we were intrigued by the finding that the amniotic membrane with limbal epithelial cells was initially opaque but became transparent after healing. For this reason, we investigated changes of extracellular matrix components during wound healing. Specifically, we analysed the expression of collagen IV (Coll-IV), a basement membrane component, which is expressed by injured corneal stromal fibroblasts. ${ }^{7}$ We also studied fibronectin (Fn), a provisional matrix component, which is expressed by stromal fibroblasts during early and active wound healing. ${ }^{8}$ Furthermore, we determined whether fibroblasts expressed $\alpha$-smooth muscle actin ( $\alpha$-SMA), a marker of myofibroblasts, ${ }^{9}$ responsible for corneal wound contraction. ${ }^{10}$ The significance of our findings is further discussed

\section{MATERIALS AND METHODS Special materials}

Monoclonal antibodies against Fn (Sakajama, Japan), CollIV (Southern Biotecnicals, AL, USA), and $\alpha$-SMA (Dako, Carpintera, CA, USA) were obtained. DAPI mounting media Vectashield was from Vector Laboratories (Burlingame, CA, USA).

\section{Rabbit model of total limbal deficiency}

The study included a total of 16 rabbits, which were a part of a study protocol that was approved by the animal research committee of the University of Miami. Two additional normal uninjured rabbits were added as normal controls. Left eyes of the 16 rabbits were rendered total limbal stem cell deficient as previously described ${ }^{11} 12$ by 1 -n-heptanol and mechanical debridement of the cornea epithelium and surgical lamellar removal of a circular rim of limbus. The state of limbal deficiency was confirmed in all these rabbits using impression cytology ${ }^{13}$ and based on a published diagnostic criterion. $^{14}$

\section{Preparation of rabbit AM, limbal biopsy, and AM culturing}

Rabbit AM was harvested from 28 day pregnant rabbits under sterile conditions, prepared and preserved as previously described. After thawing and rinsing in HBSS, the AM was fastened onto inserts. ${ }^{15}$ A limbal biopsy was obtained and cultured in the AM containing inserts as previously described. ${ }^{6}$

\section{Surgical transplantation}

Following complete pannus removal in 16 rabbits, 14 left eyes received AM transplantation with expanded epithelial cells ${ }^{6}$ 
in a manner as previously described. ${ }^{6}$ Two left eyes underwent AM alone without expanded epithelium as a control.

\section{Clinical and phenotypic outcomes}

According to the clinical outcome, each eye was classified as "success" if a smooth, avascular and clear cornea was restored; as "partial success" if more than two quadrants of the cornea was smooth, clear and avascular; and as "failure" if more than three quadrants of the cornea remained irregular, vascularised, and scarred. All corneas were then subjected to immunostaining using antibodies to cornea specific keratin $\mathrm{K}^{16}{ }^{16}$ and to conjunctival goblet cell specific MUC5AC. ${ }^{17}$ The resultant epithelium following reconstruction was defined as "corneal" if positive to $\mathrm{K} 3$ but negative to MUC5AC, as "conjunctival" if positive to MUC5AC but negative to $\mathrm{K} 3$, or as "mixed" if it was partly positive to $\mathrm{K} 3$ and partly to MUC5AC.

\section{Immunostaining}

Sections of corneoscleral buttons were fixed in cold acetone for 10 minutes at $-20^{\circ} \mathrm{C}$. After incubation in BSA to decrease non-specific staining, sections were incubated overnight with the following primary antibodies in an appropriate dilution: mouse anti-Fn (1:60), mouse anti- $\alpha$-SM (1:100), and goat anti-Coll-IV (1:50). The binding with the primary antibody was detected by a rhodamine conjugated secondary antibody. Finally, sections were mounted in anti-fainting solution containing DAPI for nuclear counterstaining.

\section{RESULTS}

\section{Follow up and clinical outcome}

All 16 experimental rabbits were followed up for 10 (SD 3.3) months (range 7-13 months). The clinical outcome of the two eyes receiving AM transplantation alone was graded as "failure" as reported. ${ }^{6}$ The remaining 14 eyes receiving ex vivo expansion were graded as "success" $(\mathrm{n}=6$, follow up of 11.8 (0.4) months), "partial success" ( $\mathrm{n}=4$, follow up of 12 (1.1) months), and "failure" $(\mathrm{n}=4$, follow up of 6.1 (0.9) months). Two normal uninjured rabbit eyes were also included as a control.

\section{Phenotypic outcome and stromal changes \\ Normal control group}

In the normal uninjured cornea, the epithelium was stratified and rested on a Bowman's membrane, and the stroma was compacted with keratocytes without vascularisation (Fig 1A). The full thickness of the corneal epithelium expressed K3 keratin (Fig lB) as reported. ${ }^{16}$ Immunostaining to Coll-IV was positive in the basement membrane of the corneal and conjunctival epithelia as a thin line, which under higher magnification was thinner in the peripheral and central cornea (Fig 1C) but thicker and wavy in the conjunctiva (Fig ID). No staining was observed in the corneal stroma. Coll-IV was also present in the basement membrane of blood vessels of the subconjunctival stroma and the Descemet's membrane (not shown). No immunoreactivity against Fn was seen in the corneal basement membrane or stroma (Fig $1 \mathrm{E}$ ), while a weak reactivity to Fn was noted in blood vessels of the conjunctiva (Fig $1 \mathrm{~F}$, indicated by a white arrow), consistent with the notion that serum Fn was present. Positive $\alpha$-SMA staining was only found in the smooth muscle of blood vessels of the conjunctiva ( similar to other groups shown below, Fig $2 \mathrm{G}$ ).

\section{Success group}

In this group, all six corneas showed a smooth and clear reconstructed surface without vascularisation (Fig 2A for an example). These corneas were associated with restoration of a corneal epithelial phenotype, which was positive to K3 keratin (Fig 2B, arrow marks the limbus), but negative to MUC5AC, which was a mucin expressed by conjunctival goblet cells (Fig 2C, arrow marks the limbus). The corneal stroma had an organised lamellar architecture and lack of inflammatory cell infiltration and vascularisation. In some cases, an eosinophilic layer of matrix, corresponding to the area where AM was applied, was noted immediately subjacent to the epithelium (not shown). Immunostaining to Coll-IV resembled what was found in the normal control. It stained a thin subepithelial basement membrane in the central and peripheral corneal areas and Descemet's membrane, but not the stroma (Fig 2D). In addition, it stained a thicker basement membrane in the conjunctival area, and the basement membrane of blood vessels in the peripheral cornea, limbal, and conjunctival stroma (Fig 2E, marked by white arrowheads). Immunostaining to Fn was negative (Fig 2F). Immunostaining to $\alpha$-SMA was positive in the smooth muscle of blood vessels of the limbal and conjunctival stroma (Fig 2G), but not in the regressing blood vessels of the peripheral cornea (Fig 2G, indicated by bracket).

Partial success group

Corneas of this group showed a smooth clear and avascular surface in one area and a hazy vascularised surface in another area (for examples see Fig 3A and F). In some corneas with partial success, the overlying epithelium was of a corneal phenotype in one side (Fig 3B, white arrowhead shows the beginning of $\mathrm{K} 3$ keratin expression), and of a conjunctival epithelium with positive expression of MUC5AC in the other side (not shown). The stroma under the corneal epithelium had a normal density of keratocytes and did not have inflammatory cells or vascularisation (not shown). In contrast, the stromal area under a conjunctival epithelium had increased intrastromal cell infiltration and was vascularised. Two rabbits in this group showed AM remnant in the corneal stroma. One of them, AM remnant was in the centre of a granuloma in the superficial stroma (Fig 3C, demarcated by asterisks). Immunostaining to Coll-IV showed a thin basement membrane in the area with a corneal epithelium, but a thick and wavy basement membrane in the area with a conjunctival epithelium (Fig 3D and G). Importantly, immunostaining to Coll-IV was clearly detected in the superficial corneal stroma of the conjunctivalised area but not in the area where the corneal epithelium was restored (Fig 3D and G, marked by arrows). Coll-IV was also found the amniotic basement membrane of AM remnant (Fig 3D, asterisk). Immunostaining to Fn was strongly positive in the anterior stroma regardless of the presence of AM (Fig 3H). Immunostaining to $\alpha$-SMA was negative when there was AM remnant (Fig 3E), but was uniformly positive in the superficial stroma when there was no AM (Fig 3I).

\section{Failure group}

All four corneas with failure following ex vivo expansion showed diffuse vascularisation and stromal haze (for an example see Fig 4A). They were covered by a conjunctival epithelium containing goblet cells with MUC5AC positive goblet cells (Fig 4B), but negative to K3 keratin (not shown). The underlying stroma showed loss of lamellar structure and was infiltrated with blood vessels. Histological sections showed that AM could not be identified in all corneas (not shown). Strong immunostaining to Coll-IV was found in the basement membrane (Fig 4C), and in blood vessels that infiltrated in the corneal stroma (Fig 4C and inset showing high magnification). Immunostaining to Fn was negative in the corneal stroma of all failure corneas (Fig 4D). Immunostaining to $\alpha$-SMA was not seen in the corneal stroma nor in infiltrating blood vessels (Fig 4E). 


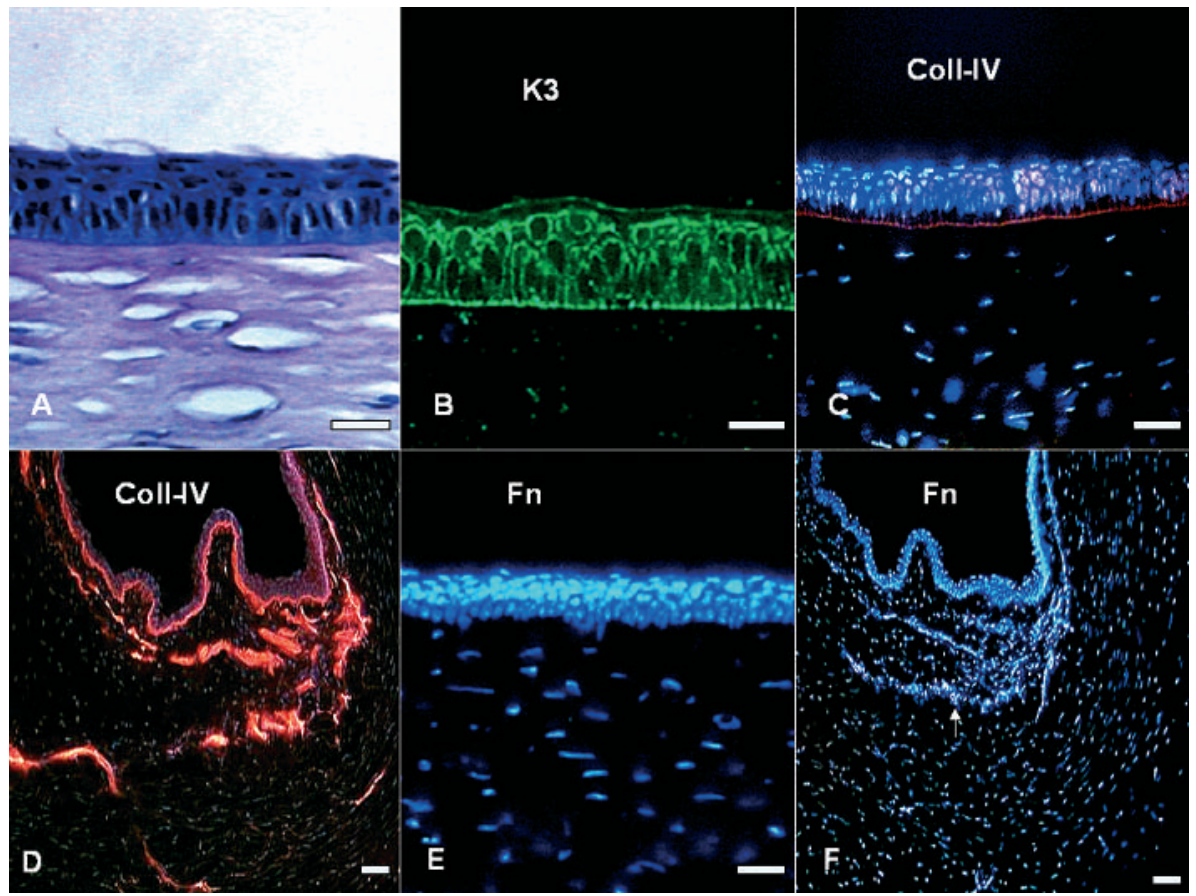

Figure 1 Normal control cornea.

Haematoxylin staining (A). Keratin 3

(K3) is expressed in the full thickness of the corneal epithelium (B). Collagen IV staining in the basement membrane of corneal epithelium was weak and thin (C) while thicker and wavy in the conjunctival region and the underlying stromal blood vessels (D). Fibronectin

$(\mathrm{Fn})$ staining was negative in the entire cornea (E) and conjunctiva (F) except for the lumen of blood vessels (arrow). Bar $50 \mu \mathrm{m}$.

\section{Failure following AMT alone}

The two corneas that failed after AM transplantation alone also showed diffuse vascularisation and stromal haze (for an example see Fig 5A). They were covered by a conjunctival epithelium containing goblet cells with MUC5AC positive goblet cells (not shown). The underlying stroma showed loss of lamellar structure and was infiltrated with blood vessels. Histological sections showed that AM remnants could be found in one cornea (Fig 5A, B, and D, demarcated by brackets). Strong immunostaining to Coll-IV was found in the subepithelial basement membrane, the subepithelial region corresponding to $\mathrm{AM}$ remnant, and superficial stroma subjacent to or near AM (Fig 5C and D, marked by arrows). Immunostaining to Fn was negative in AM remnant, but was positive in the epithelial basement membrane, superficial stroma, and subjacent blood vessels of the area near AM remnant (Fig 5E, marked by asterisk). Immunostaining to $\alpha$ SMA was negative in the entire stroma including infiltrating blood vessels (Fig 5F).

\section{DISCUSSION}

The ex vivo expansion of limbal stem cells is a new technique that may help restore corneal transparency in patients with total limbal SC deficiency. This report together with our earlier one ${ }^{6}$ clearly illustrates its potential efficacy in restoring the normal corneal phenotype and promoting stromal wound healing and remodelling, leading to transparency in a rabbit model with total limbal SC deficiency.

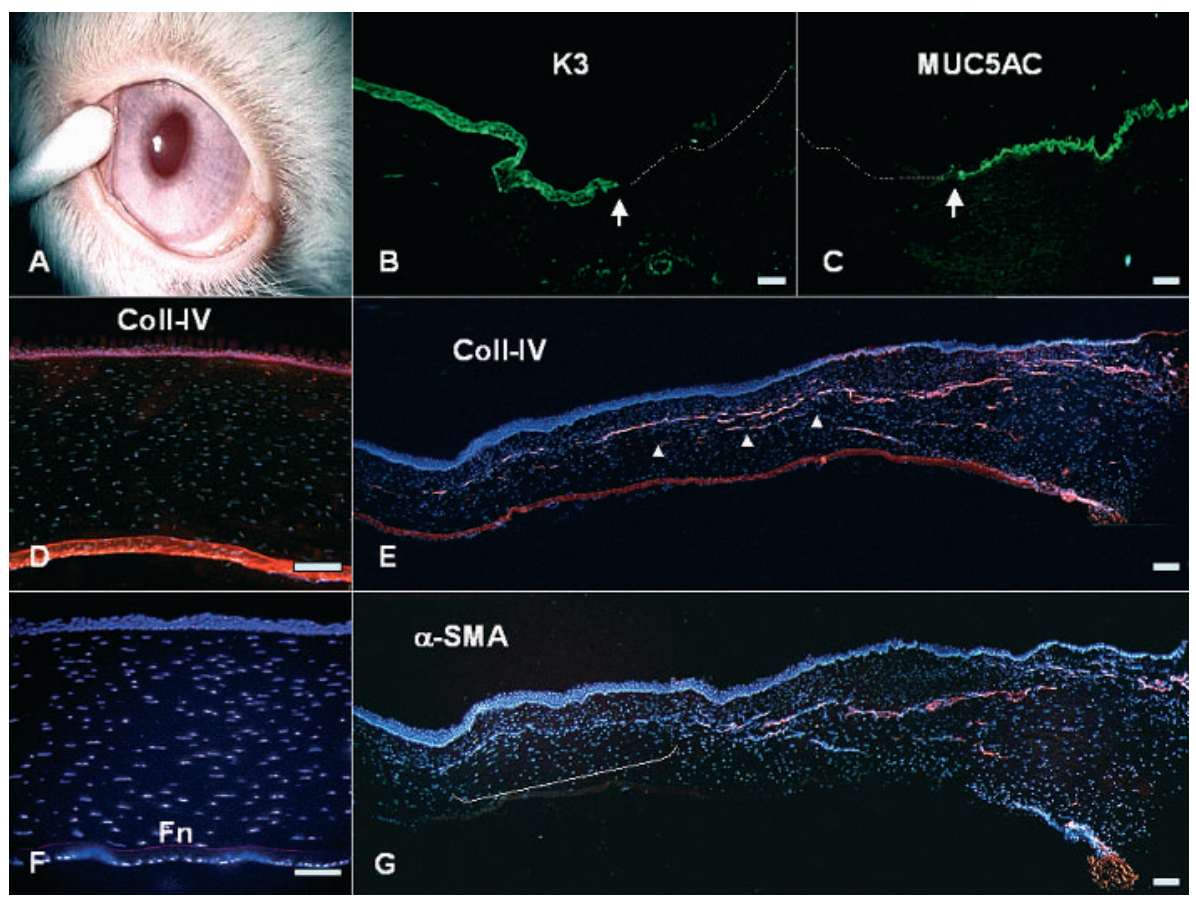

Figure 2 Cornea with success. External appearance shows that the reconstructed surface is smooth and clear without vascularisation (A). Keratin $3(\mathrm{~K} 3)$ is expressed in the reconstructed corneal epithelium (B, arrow marks the limbus). MUC5AC positive goblet cells are limited to the conjunctival region $(C$, arrow marks the limbus). Coll-IV is expressed in the corneal basement membrane and Descemet's membrane but not in the stroma (D). Coll-IV is also expressed in the stroma of peripheral cornea and limbal region (E). Immunoreactivity against fibronectin $(\mathrm{Fn})$ was negative (F). $\alpha$-SMA was expressed in the smooth muscle of blood vessels of the limbal and conjunctival stroma (G). Bar $50 \mu \mathrm{m}$. 


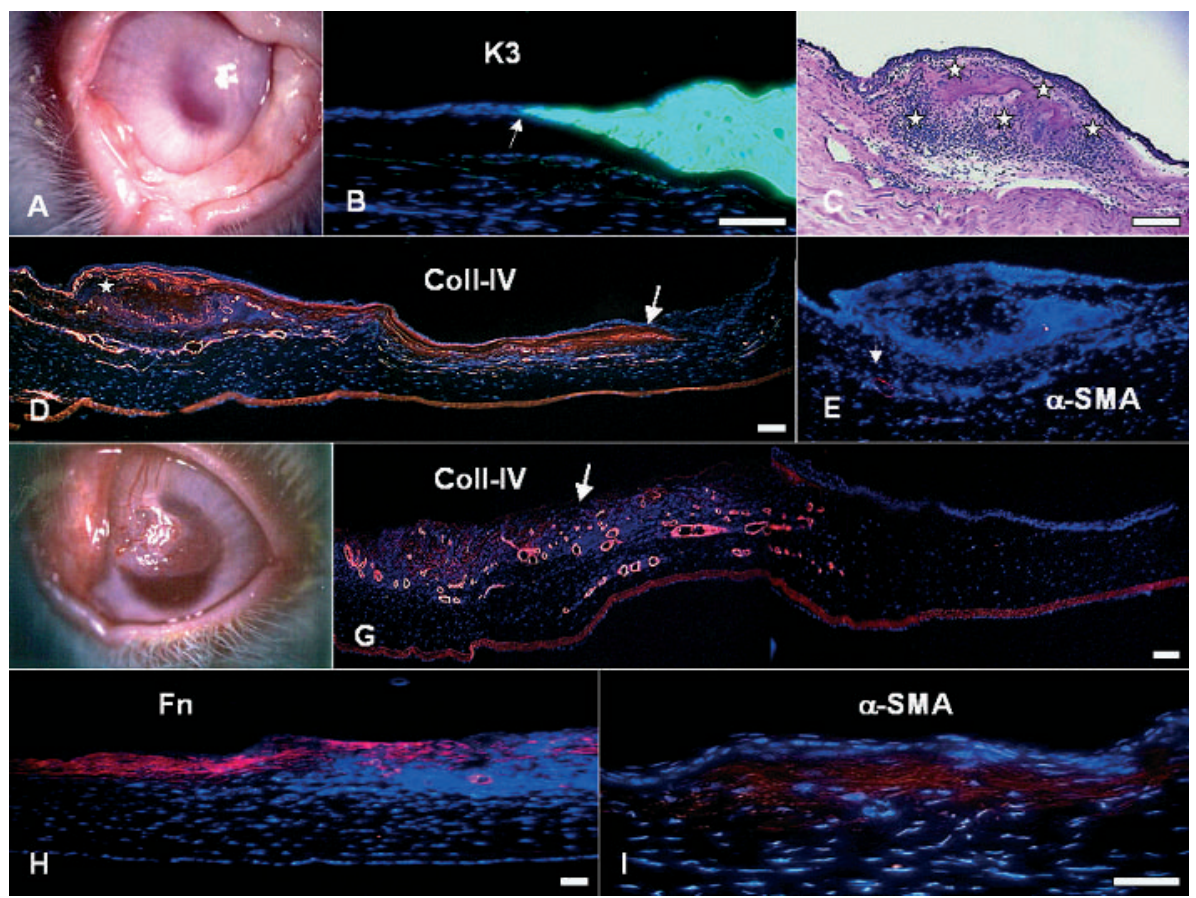

Figure 3 Corneas with partial success. Slit lamp appearance shows that part of these corneas is smooth and clear and the other is vascularised and opaque

$(A, F)$. Keratin $3(K 3)$ is expressed only in the smooth and clear area (arrow marks the border between corneal and conjunctival phenotypes) (B).

Haematoxylin and eosin staining shows AM remnant (marked by asterisks) (C). Coll-IV is expressed in the corneal basement membrane, AM remnants and the superficial corneal stroma (D). $\alpha$-SMA was only expressed in the smooth muscles of blood vessels in the limbal stroma (arrowhead marks a blood vessel) (E). Coll-IV is expressed in superficial stroma and intrastromal blood vessels (G). Fibronectin (Fn) was highly expressed in the stroma and blood vessels of granuloma (H). $\alpha$-SMA expression suggests the presence of myofibroblasts in the granuloma (I). Bar $50 \mu \mathrm{m}$.

After a long term follow up of 11.8 (0.4) months, we have confirmed that a normal stromal architecture with the lack of Coll-IV and Fn deposition, identical to that of normal corneas, was restored in those corneas with successful restoration of a corneal epithelial phenotype. Similarly, a normal stromal architecture was also restored in the area with a corneal epithelium but lost in the area with conjunctival epithelium of those corneas with partial success. In the latter area, the superficial stroma was deposited with Coll-IV and Fn. Deposition of Coll-IV was also found in rabbit corneal stroma injured by alkali burns or laceration, was associated with activated stromal fibroblasts, and was thought to be the reason for forming opaque granulation tissue. $^{7}$ In the early stage of wound healing, fibronectin exudated from the plasma or tears and secreted by activated fibroblasts is deposited in the corneal stroma as a provisional matrix. Fn is completely reabsorbed when the wound healing process is completed. ${ }^{18}$ We thus surmise that wound remodelling remained incomplete in corneas with partial success. To our surprise, we did not observe deposition of Coll-IV or Fn in the corneal stroma of the failure group no matter whether the failure resulted from ex vivo expansion or not. Therefore, we conclude that continuous wound remodelling is not associated with the presence of a conjunctival epithelium.

That was the reason why we also looked into the presence of myofibroblasts, which characteristically express contractile $\alpha$-SMA in cytoskeletons and integrin $\alpha 5 \beta 1$ on cytoplasmic

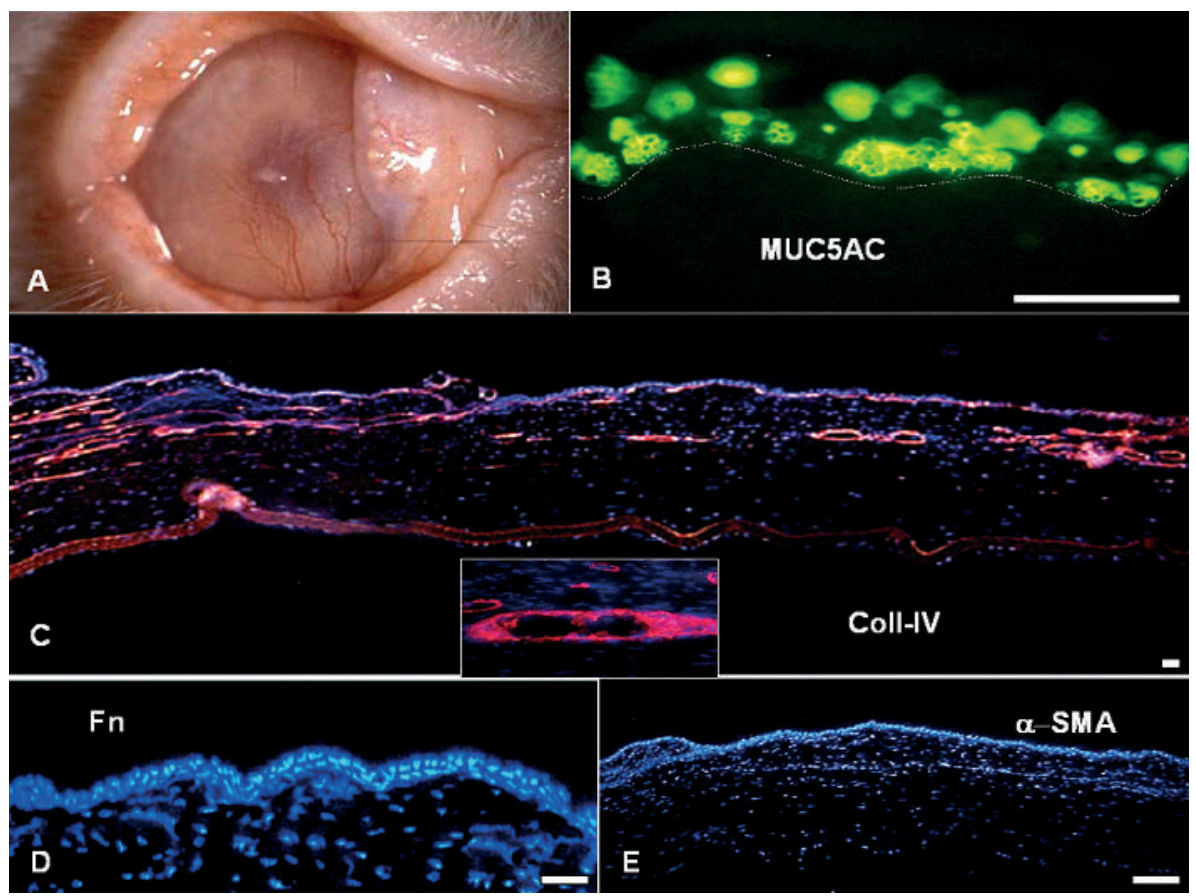

Figure 4 Cornea with failure following ex vivo expansion. Slit lamp appearance shows corneal haze, vascularisation, and inflammation $(A)$ MUC5AC staining of the goblet cells confirms conjunctival invasion of the corneal surface (B). Coll-IV is expressed in the corneal basement membrane and intrastromal blood vessels. Inset shows the higher magnification of blood vessel wall (C). Fibronectin (Fn) and $\alpha$-SMA were expressed only in stromal blood vessels ( $D$ and $E$, respectively). Bar $50 \mu \mathrm{m}$. 


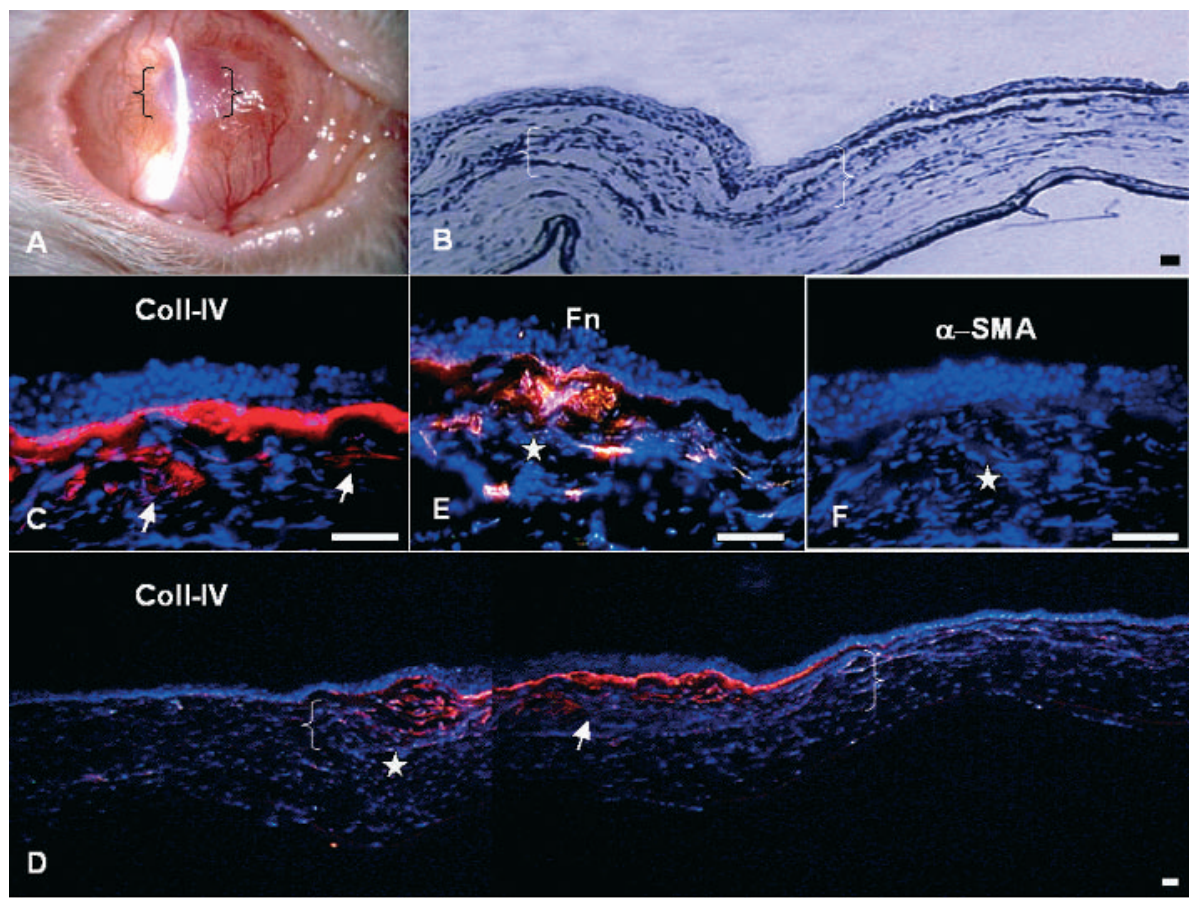

Figure 5 Cornea with failure following AM transplantation alone. Slit lamp appearance shows corneal haze and vascularisation. $A M$ remnants are seen in the stroma (enclosed by brackets) (A). Haematoxylin stain shows the presence of $A M$ remnants in the cornea (enclosed by brackets) (B). Coll-IV is highly expressed in the corneal basement membrane and stroma (marked by arrows) (C). Coll-IV is also found in AM remnants (enclosed by brackets) (D). (Fibronectin (Fn) is also found in the corneal stroma with AM remnants (E). $\alpha$-SMA is not expressed in this area (F). Bar $50 \mu \mathrm{m}$.

membranes, and deposit EDA domain containing cellular fibronectin in the pericellular matrix, and collagens and proteoglycans in the stromal matrix (for reviews see Jester et $\left.a l^{10}\right)$. In this report, we noted that expression of $\alpha$-SMA was absent in the stromal fibroblasts in corneas with successful reconstruction, similar to that of normal corneas. In contrast, $\alpha$-SMA positive myofibroblasts were found in the same area deposited with Coll-IV and Fn in corneas with partial success. This result supports the notion that complete remodelling is associated with the absence of myofibroblasts, and that incomplete remodelling leads to the persistence of myofibroblasts. Again, we did not detect any myofibroblasts in failure corneas, further confirming that corneal stromal opacity in failure corneas was not the result of myofibroblasts. Besides the reason that a conjunctival epithelium covering the cornea is not optically as sound as the corneal epithelium, the opacity in failure corneas might also be caused by intense infiltration of blood vessels. These blood vessels contained Coll-IV (in the basement membrane) but not $\alpha$-SMA (which is also found in smooth muscle cells), indicating that they were capillaries (Figs 3-5). The other contributing factor to stromal opacity might be infiltration of chronic inflammatory cells.

It is intriguing to note that expression of $\alpha$-SMA in corneas with partial success was aborted in the area with AM remnant (Fig 3). This finding suggests that formation of myofibroblasts is prohibited by AM, a finding consistent with our previously reports showing that myofibroblasts are inhibited by AM in vivo and in vitro ${ }^{19}$ and that TGF $\beta$ (tumour necrosis factor $\beta$ ) signalling and myofibroblast differentiation are downregulated by AM stroma. ${ }^{20} 21$ Taken together, we believe investigations into the mechanism whereby corneal stromal transparency can be achieved by restoration of a corneal epithelial phenotype and complete remodelling of AM will unveil additional applications in the future.

\section{ACKNOWLEDGEMENTS}

Supported by Public Health Service Research Grant EY06819 (to SCGT via TissueTech, Inc) from Department of Health and Human Services, National Eye Institute, National Institute of Health, Bethesda, MD, USA; Human Manpower Development Program, Singapore National Eye Centre, Singapore (to SET), and research fellowship grant GR-1814/1-2 (to MG) by the Deutsche Forschungsgemeinschaft, Bonn, Germany, and a grant from Ocular Surface Research \& Education Foundation, Miami, FL, USA.

\section{Authors' affiliations}

E M Espana, M Grueterich, A Touhami, S C G Tseng, TissueTech, Inc. and Ocular Surface Center, Miami, FL, USA S-E Ti, Singapore National Eye Centre, Singapore

Proprietary interest: SCGT and his family are more than $5 \%$ shareholders of TissueTech, Inc, which owns US Patent Nos 6,152,142 and $6,326,019$ on the method of preparation and clinical uses of human amniotic membrane, distributed by Bio-Tissue, Inc.

Presented in part at the annual meeting of ARVO in Ft Lauderdale, $\mathrm{FL}$, USA, in May 2003.

\section{REFERENCES}

1 Tsai RJF, Li L-M, Chen J-K. Reconstruction of damaged corneas by transplantation of autologous limbal epithelial cells. N Eng J Med 2000;343:86-93.

2 Schwab IR, Reyes M, Isseroff RR. Successful transplantation of bioengineered tissue replacements in patients with ocular surface disease. Cornea 2000;19:421-6

3 Koizumi N, Inatomi T, Suzuki T, et al. Cultivated corneal epithelial transplantation for ocular surface reconstruction in acute phase of StevensJohnson syndrome. Arch Ophthalmol 2001;119:298-300.

4 Koizumi N, Inatomi T, Suzuki T, et al. Cultivated corneal epithelial stem cell transplantation in ocular surface disorders. Ophthalmology 2001; 108:1569-74.

5 Grueterich M, Espana EM, Touhami A, et al. Phenotypic study of a case with successful transplantation of ex vivo expanded human limbal epithelium for unilateral total limbal stem cell deficiency. Ophthalmology 2002; 109:1547-52.

6 Ti S-E, Anderson DF, Touhami A, et al. Factors affecting outcome following transplantation of ex vivo expanded limbal epithelium on amniotic membrane for total limbal deficiency in rabbits. Invest Ophthalmol Vis Sci 2002;43:2584-92.

7 Ishizaki M, Shimoda M, Wakamatsu K, et al. Stromal fibroblasts are associated with collagen IV in scar tissues of alkali-burned and lacerated corneas. Curr Eye Res 1997;16:339-48.

8 Garana RMR, Petroll WM, Chen WT. Radial keratomy II: role of the myofibroblast in corneal wound contraction. Invest Ophthalmol Vis Sci 1992:33:3271-82.

9 Gabbiani G, Chaponnier C, Hüttner I. Cytoplasmic filaments and gap junctions in epithelial cells and myofibroblasts during wound healing. I Cell Biol 1978;76:561-8.

10 Jester JV, Petroll WM, Cavanagh HD. Corneal stromal wound healing in refractive surgery: the role of myofibroblasts. Prog Retin Eye Res $1999 ; 18: 311-56$. 
11 Kruse FE, Chen JJY, Tsai RJF, et al. Conjunctival transdifferentiation is due to the incomplete removal of limbal basal epithelium. Invest Ophthalmol Vis Sci 1990;31:1903-13.

12 Huang AJW, Tseng SCG. Corneal epithelial wound healing in the absence of limbal epithelium. Invest Ophthalmol Vis Sci 1991;32:96-105.

13 Tseng SCG. Staging of conjunctival squamous metaplasia by impression cytology. Ophthalmology 1985;92:728-33.

14 Puangsricharern V, Tseng SCG. Cytologic evidence of corneal diseases with limbal stem cell deficiency. Ophthalmology 1995;102:1476-85.

15 Meller D, Tseng SCG. Conjunctival epithelial cell differentiation on amniotic membrane. Invest Ophthalmol Vis Sci 1999;40:878-86.

16 Schermer A, Galvin S, Sun T-T. Differentiation-related expression of a major $64 \mathrm{~K}$ corneal keratin in vivo and in culture suggests limbal location of corneal epithelial stem cells. J Cell Biol 1986;103:49-62.
17 Kim JC, Tseng SCG. Transplantation of preserved human amniotic membrane for surface reconstruction in severely damaged rabbit corneas. Cornea 1995; 14:473-84.

18 Hendricks RL. An immunologist's view of herpes simplex keratitis. Cornea 1997:16:503-6.

19 Choi TH, Tseng SCG. In vivo and in vitro demonstration of epithelial cellinduced myofibroblast differentiation of keratocytes and an inhibitory effect by amniotic membrane. Cornea 2001;20:197-204.

20 Li D-Q, Tseng SCG. Differential regulation of keratinocyte growth factor and hepatocyte growth factor/scatter factor by different cytokines in cultured human corneal and limbal fibroblasts. J Cell Physiol 1997;172:361-72.

21 Lee S-B, Li D-Q, Tan DTH, et al. Suppression of TGF- $\beta$ signaling in both normal conjunctival fibroblasts and pterygial body fibroblasts by amniotic membrane. Curr Eye Res 2000;20:325-34.

\section{Video reports}

To view the video reports in full visit our website www.bjophthalmol.com and click on the link to video reports.

- Feeder vessel treatment with high speed ICG angiography. D Stanescu-Segall, $G$ Coscas, F Coscas, G Soubrane

- Endoscopy to aid anterior segment surgery. J E Moore, A Sharma

- Penetrating ocular injury due to a fish hook: surgical removal. SDM Chen, D Chiu, C K Patel

- Retinal ganglion cell axon response to guidance molecules $S F$ Oster, D W Sretavan

- Marin-Amat syndrome A Jogiya, C Sandy

- Excision of subcutaneous dirofilariasis of the eyelid D Mallick, T P lttyerah

- Thixotropy: a novel explanation for the cause of lagophthalmos after peripheral facial nerve palsy. M Aramideh, J H T M Koelman, P P Devriese, F VanderWerf, J D Speelman

- Surgical revision of leaking filtering blebs with an autologous conjunctival graft. K Taherian, A Azuara-Blanco

- Dipetalonema reconditum in the human eye. $T$ Huynh, $J$ Thean, $R$ Maini

- Evaluation of leucocyte dynamics in mouse retinal circulation with scanning laser ophthalmoscopy. H XU, A Manivannan, G Daniels, J Liversidge, P F Sharp, J V Forrester, I J Crane

- An intraocular steroid delivery system for cataract surgery. D F Chang

- Pearls for implanting the Staar toric IOL. D F Chang

- Capsule staining and mature cataracts: a comparison of indocyanine green and trypan blue dyes. D F Chang 